\title{
Rabelais à la jonction de deux fables (XII, 5-6) de La Fontaine
}

\section{Alessandra Marangoni}

\section{(2) OpenEdition}

1 Journals

\section{Édition électronique}

URL : http://journals.openedition.org/studifrancesi/35568

DOI : $10.4000 /$ studifrancesi.35568

ISSN : 2421-5856

Éditeur

Rosenberg \& Sellier

\section{Édition imprimée}

Date de publication : 1 juillet 2005

Pagination : 61-64

ISSN : 0039-2944

\section{Référence électronique}

Alessandra Marangoni, « Rabelais à la jonction de deux fables (XII, 5-6) de La Fontaine », Studi Francesi [En ligne], 145 (XLIX | I) | 2005, mis en ligne le 30 novembre 2015, consulté le 18 avril 2021. URL: http://journals.openedition.org/studifrancesi/35568; DOI : https://doi.org/10.4000/studifrancesi. 35568

\section{(c) (i) $\odot$}

Studi Francesi è distribuita con Licenza Creative Commons Attribuzione - Non commerciale - Non opere derivate 4.0 Internazionale. 


\section{Rabelais à la jonction de deux fables (XII, 5-6) de La Fontaine}

Les récentes lectures des Fables de La Fontaine ont été sensibles à la place de chaque fable à l'intérieur du livre qu'elle contribue à former: ordre non casuel qui lie l'une à l'autre des «monades» ${ }^{1}$ - la désignation de Spitzer n'en perd aucunement de sa validité - formant, dans leur ensemble, l'«architecture» de l'«édifice»² des Fables; construction par ailleurs en labyrinthe, ${ }^{3}$ pour les nombreux échos d'un livre à l'autre, indices d'un parcours non linéaire ${ }^{4}$.

Fort fructueusement, l'américain Randolph Paul Runyon a élu à principe de méthode cette tendance, s'appuyant notamment sur la succession d'une fable à l'autre et allant jusqu'à lire chaque texte des douze livres des Fables à la lumière et de celui qui le précède et de celui qui le suit's

Or, une lecture conjointe de deux fables - 5, 6 - se côtoyant dans celui qu'on est convenu d'appeler le douzième livre ${ }^{6}$ (septembre 1693) des Fables, nous a mis en présence d'une nouvelle source de l'apologue "Le Cerf malade" (XII, 6). Fait qui pourrait être, en soi, secondaire, voire négligeable, si la source n'était le Tiers Livre de Rabelais $^{7}$ et si elle ne devenait exemplaire d'une modalité de transition d'un poème à l'autre. Goutte dans la mer pourtant, parce qu'intertexte ne fonctionnant qu'à la charnière de deux apologues sur deux cent cinquante.

Nous avons dit «source». Il vaut mieux parler de réminiscence textuelle éclairant la proximité de deux textes, voire imposant un ordre séquentiel à deux d'entre les fables.

(1) L. SPITZER, L'Art de la transition chez La Fontaine [1938, 1959] in Etudes de style, Paris, Gallimard, 1970, p. 197. J.-P. Collinet, La Rochefoucault et La Fontaine, «Littératures », n. 15, 1986, p. 73.

(2) «Les Fables possèdent une architecture secrète. Un double-fond. Un ordre qui soutient l'édifice.» A.-M. BAssy, Introduction à LA FoNTAINe, Fables, GF/Flammarion, 1995, p. 30. En 1952, Wadsworth (Young La Fontaine) supposait une «architecture» aux fables. Plusieurs spécialistes se sont, depuis, essayés à en faire ressortir les lignes de force, soient-elles thématiques (P. BORNECQUE, «Thèmes et organisation des fables», Europe, mars 1972, pp. 39-52; G. CouTON, Le livre épicurien des Fables: essai de lecture du livre VIII, «Travaux de linguistique et de littérature», XIII, 2, 1975, pp. 283-290; A. Niderst, Sur la composition des Fables de La Fontaine, «The French Review», n. 2, december, 1991, pp. 187-194), soient-elles structurelles (J. PROUST, Remarques sur la disposition par livres des Fables de La Fontaine, in De Jean Lemaire de Belges à Jean Giraudoux. Mélanges d'bisoire et de critique littéraire offerts à Pierre Jourda, Paris, Nizet, 1970 , pp. 227-248; M. SLATER, La Fontaine's Fables, book VII: the problem of order, «The Modern Language Review», n. 3, july 1987; J. LAFOND, L'architecture des livres VII à XII des Fables, «Le Fablier», n. 4, 1992).

(3) Pour le recours à l'image du labyrinthe - idée complémentaire et non opposée à celle de séquence linéaire -, cf. L. SPITZER, cit., p. 196, A.- M. BASSY, Les Fables de La Fontaine et le Labyrinthe de Versailles, «Revue française d'histoire du livre», n. 12, 1976, pp. 367-426 (étude dont on trouve un résumé à l'article "La Fontaine" du Dictionnaire des Littératures de langue française, Bordas, 1987), R. DANNER, La Fontaine's Fables, Book. X: the Labyrinth Hypothesis, 1981, vol. XXI, n. 4, pp. 90-98.

(4) P. Dandrey parle de Le cordeau et le hasard: réflexions sur l'agencement du recueil des Fables, «Papers on French Seventeenth Century Literature», n. 44, 1996, pp.73-85 (actes du colloque international La Fontaine de Londres, 17-18 févr. 1995).

(5) R. P. Runyon, In La Fontaine's Labyrinth: a Thread through the Fables, Charlottesville, Roowood Press, 2000. N. Gross avait ouvert la voie avec sa lecture du livre VI: Order and Theme in La Fontaine's Fables, Book VI, L'Ésprit Créateur, 1981, vol. XXI, n. 4, pp. 78-89.

(6) Voir, à ce propos, les précisions de $\mathrm{PH}$. A. WADSWORTH, Le Douzième livre des fables, «Cahiers de l'Association Internationale des Études Françaises», n. 26, mai 1974, p. 104.

(7) «maître François, dont je me dis encore le disciple». À M. de Saint-Évremond (lettre de décembre 1687), J. DE La Fontaine, Euvres complètes, t. II, édition de P. Clarac, "Pléiade"/Gallimard, 1958, p. 674. 
On se souviendra du chat de la fable XII, 5 "Le vieux Chat et la jeune Souris", chat qui a nom Raminagrobis ${ }^{8}$ (v. 3), comme déjà, par ailleurs, le chat figurant dans VII, 15 "Le Chat, la Belette et le petit Lapin" (exemple flagrant d'écho à distance):

XII, 5

\section{LE VIEUX CHAT ET LA JEUNE SOURIS}

Une jeune Souris de peu d'expérience

Crut fléchir un vieux Chat implorant sa clémence,

Et payant de raisons le Raminagrobis :

Laissez-moi vivre : une Souris

De ma taille et de ma dépense

Est-elle à charge en ce logis ?

Affamerais-je, à votre avis,

L'Hôte et l'Hôtesse, et tout leur monde?

D'un grain de blé je me nourris ;

Une noix me rend toute ronde.

A présent je suis maigre; attendez quelque temps

Réservez ce repas à Messieurs vos Enfants.

Ainsi parlait au Chat la Souris attrapée.

L'autre lui dit: Tu t'es trompée.

Est-ce à moi que l'on tient de semblables discours?

$\mathrm{Tu}$ gagnerais autant de parler à des sourds.

Chat et vieux pardonner ? cela n'arrive guères.

Selon ces lois descends là-bas,

Meurs, et va-t'en tout de ce pas

Haranguer les soeurs Filandières.

Mes Enfants trouveront assez d'autres repas.

Il tint parole; et, pour ma fable,

Voici le sens moral qui peut y convenir :

La jeunesse se flatte, et croit tout obtenir.

La vieillesse est impitoyable.

On se souviendra également de la situation décrite dans la fable suivante, la sixième du livre XII, "Le Cerf malade", dont la source reconnue est une fable de Lokman ${ }^{10}$

(8) Le premier à associer ce nom - dans sa variante de Rominagrobis - à un chat est Voiture (lettre à l'abbesse d'Yères): Euvres, éd. Ubicini, 1855, Slatkine Reprints, 1967, p. 424. L'ascendance du mot a été d'abord reconstruite par $\mathrm{H}$. Régnier dans son édition (Grands Écrivains de la France/Hachette) des Euvres de La Fontaine (t. II, 1884, pp. 187-188). Collinet y ajoute, parmi les écrivains du XVII ${ }^{\mathrm{e}}$ s., la référence à Sarasin.(J. DE La Fontaine, Euvres complètes, t. I, "Pléiade"/ Gallimard, 1991, p. 1184. Edition d'où nous citerons les fables.)

(9) Relativement à l'incidence du personnage de Raminagrobis sur le septième livre des Fables, cf. M. SLATER, art. cit., p. 576.

(10) Ou Luqman, personnage cité dans le Coran. $\mathrm{La}$ légende lui attribue une quarantaine de fables qui sont une version arabe de fables d'Esope.

(11) «que la Fontaine pouvait connaître par la traduction latine d'Erpenius (1615), ou par la traduction en vers latins de Tanaquil Faber», G. Couton, édition de La Fontaine, Fables, Garnier
Frères, 1962, p. 538. Voici la version d'Erpenius et les vers qu'en tire Tanaquil Faber (Tanneguy Lefèvre): «Fab. III /Cervvs/ Thom. ERPEN. / Cervus aliquando cum agrotaret, ad cum venerunt Socii eius de feris, et custodiverunt eum, graminaque et berbas, que circum cum erant absumpserunt. Is autem eum convaluisset, quasivit quod ederet, nec invenit quicquam. unde fame periit./ Hoc significat / Multiplicari dolores eius, cuius familia multiplicatur. // TAN. FABER / Cervus iacebat morbo decumbens gravi: / Extemplo ad illum totius viciniae / Cohors fidelis, Quadrupedes, pium genus, / Venere, dictis si dolorem averterent: I Et cuncta Cervi consumpsere gramina. I Sed ipse paucos cum coepisset post dies / Revocate notas fessi vires corporis / Circaque nudum late conspiceret solum. / Verbis dolorem sic testatus est suum: / Valete, amici, vestrum officium nil moror: / Solatiorum abunde est, pabuli nibil // Tales videmus seclo nostro plurimos, I Qui verba nos afferunt, et rem harpagant./ Domesticorum populus adducit famem, / Et inaniarum plena reddit borrea. (Tanaquili Fabri Fabulae ex Locmanis arabico latinis 
("La Gazelle"11), actualisée, en 1677, par L.-S. Desmay ("Le Cerf malade ou la grande alliance nuisible" ${ }^{12}$ ):

\author{
XII, 6 \\ LE CERF MALADE
}

En pays plein de Cerfs un Cerf tomba malade.

Incontinent maint Camarade

Accourt à son grabat le voir, le secourir,

Le consoler du moins; multitude importune.

Eh! Messieurs, laissez-moi mourir.

Permettez qu'en forme commune

La Parque m'expédie, et finissez vos pleurs.

Point du tout : les Consolateurs

De ce triste devoir tout au long s'acquittèrent ;

Quand il plut à Dieu s'en allèrent.

Ce ne fut pas sans boire un coup,

C'est-à-dire sans prendre un droit de pâturage.

Tout se mit à brouter les bois du voisinage.

La pitance du Cerf en déchut de beaucoup

Il ne trouva plus rien à frire

D'un mal il tomba dans un pire,

Et se vit réduit à la fin

Â jeûner et mourir de faim.

Il en coûte à qui vous réclame,

Médecins du corps et de l'âme.

Ô temps, ô moeurs! J'ai beau crier,

Tout le monde se fait payer.

Or, la contiguïté des deux fables ne saurait être mieux expliquée que par le personnage de Raminagrobis mourant, dont voici les mots tirés du vingt et unième chapitre du Tiers Livre:

Allez, enfans, en la garde du grand Dieu des cieux, \& plus de cettui affaire ne d'autre que soit, ne m'inquietez. J'ay ce jourd'hui, qui est le dernier de May \& de moy, hors ma maison en grande fatigue $\&$ difficulté, chassé un tas de vilaines, immondes, \& pestilentes bêtes noires, garres, fauves, blanches, cendrées, grivolées, lesquelles laisser ne me vouloient à mon aise mourir, \& par fraudulentes pointures, gruppemens harpiaques, importunitez frêlonniques, toutes forgées en l'officine de ne sçay quelle insatiabilité, me évoquoient du doux pensement, auquel j'acquesçois [...]; plus ne me molestez, \& me laissez en silence, je vous supplie. ${ }^{13}$

Réminiscence activée par le nom de Raminagrobis dans la première des deux fables et agissant non seulement sur leur ordonnance, mais aussi sur le vocabulaire de la deuxième, dont on n'est pas sans remarquer les reprises lexicales «multitude importune» et «laissez-moi mourir» (cette dernière, par ailleurs, en évident rapport d'oppositon spéculaire avec le «laissez-moi vivre» du texte précédent ${ }^{14}$ ). Imploration

versibus redditae, Salmurii, Apud Renatum Pean, 1673, pp. 8-10.)

(12) L'adaptation de Desmay (Ésope du temps, fable V) est reproduite dans l'édition d'A. VERSaIlle: La Fontaine, Euvres. Sources et Postérité d'Ésope à l'Oulipo, préf. de M. Fumaroli, Paris, Editions Complexe, 1995, pp. 1417-18.

(13) Les ouvres de M. François Rabelais Docteur en Medecine. Augmentées de la Vie de l'Auteur, E de quelque Remarques sur sa Vie E sur l'Histoire. Avec la Clef E l'explication de tous les mots dificiles. Tome premier, A Amsterdam, Chez Adrien Moetians, à la Librairie Françoise, M. DC LIX., p. 381. Nous soulignons.

(14) Cf. R. P. Runyon, étude citée, p. 164. 
dont force est de constater: «this will to death does not appear in the fables's source» ${ }^{15}$ et que, seule, la liaison textuelle pourvue par le Tiers Livre permet d'éclairer.

Point n'est besoin d'en appeler à l'idéologie: d'un côté le vieux Raminagrobis agonisant molesté par des bêtes-[moines $]^{16}$ désireuses de le convertir, quittes à en obtenir une «pitance» accrue ${ }^{17}$, de l'autre le "Cerf malade" privé de sa «pitance» (v. 14) par des «Médecins du corps et de l'âme» ${ }^{18}(v .20)$ tenus responsables, selon l'allusion qu'entrevoyait G. Couton, d'une conversion arrachée sur le lit de mort $^{19}$. Mais glissement tentant si l'on considère que la fable "Le vieux Chat et la jeune Souris" vient de rectifier l'assimilation provisoire du fabuliste à la souris - équation jeune Prince : ma Muse $=$ Chat : Souris que posait le prologue à la fable proprement dite ${ }^{20}$ à travers l'analogie tacite entre le vieux fabuliste et le vieux chat nommé Raminagrobis.

Morceau textuel, celui venant du vingt et unième chapître du Tiers Livre, œvrant de manière manifeste dans le nom du chat de la fable 5 ainsi que dans le choix lexical et situationnel de la fable 6 et, de manière implicite - in absentia: dans l'entre-deux -, mais non moins puissament, sur leur agencement. Clé qu'offre une pièce (XII, 5) pour accéder à la pièce contiguë (XII, 6), après avoir invité à revenir sur ses pas (pont intratextuel vers le Raminagrobis de VII, 15).

Non pas source de la fable 5 , ni de la fable 6, la réminiscence textuelle issue de Rabelais s'avère être à la base - la base - de leur rapport de proximité.

ALESSANDRA MARANGONI

Appendice: externe à l'édifice des Fables, un troisième Raminagrobis se voit dans «La Ligue des rats», fable parue anonyme dans le Mercure Galant de décembre 1692. Son attribution à La Fontaine demeure douteuse. Or la Loi des Mots - double occurrence de Raminagrobis dans la courte période qui doit aller de la fin de 1692 au début de 1993 - ne ferait-elle pas pencher la balance du côté de La Fontaine?

(15) ibidem

(16) «Les bêtes que chasse le mourant sont des moines». Note de F. Joukowski à Rabelais, Le Tiers Livre, GF/Flammarion, 1993, p. 276. C'était déjà l'interprétation de Screech (Le Tiers Livre, Droz, 1964, p. 153).

(17) Cf. les mots de Panurge: «Au moins s'il perd le corps \& la vie, qu'il ne damne son âne. Nous l'induirons à contrition de son peché: à requerir pardon és dits tant beats Peres [...] afin qu'aprés son trépas ils ne le déclarent heretique \& damné [...] Et que au jour de son trépas sempiternellement, ils ayent tous quintuple pitance». Les auvres de M. François Rabelais...cit., p. 384 (Tiers Livre, ch. XXIII). Sur la leçon âne pour âme, cf. édition Schreech.

(18) Cf. «Les Thelogiens à l'ame, les Medecins au corps, les Jurisconsultes aux biens» chez Rabelais, Tiers Livre, ch. XXIX. Éd. citée, p. 409.

(19) «en décembre 1692, La Fontaine, tombé malade, a reçu les médecins du corps; celui de l'âme aussi: l'abbé Pouget, qui le ramène à Dieu, lui fait abjurer ses Contes devant une délégation de l'Académie (12 février 1693).» G. CouTON, édition citée, p. 538.

(20) «Le jeune Prince alors se jouerait de ma Muse / Comme le Chat de la Souris.» À Monseigneur le duc de Bourgogne, qui avait demandé à $M$. de La Fontaine une fable qui fût nommée «Le Chat et la Souris», vv. 21-22. L'assimilation provisoire à la souris est demandée par le rang de l'élève; la relation habituelle maître-élève en est d'ailleurs renversée. 